\title{
Mice lacking inhibitory leptin receptor signals are lean with normal endocrine function
}

\author{
Marie Björnholm,1,2 Heike Münzberg, ${ }^{1}$ Rebecca L. Leshan, ${ }^{1}$ Eneida C. Villanueva, ${ }^{1}$ \\ Sarah H. Bates, ${ }^{1}$ Gwendolyn W. Louis, ${ }^{1}$ Justin C. Jones, ${ }^{1}$ Ryoko Ishida-Takahashi, ${ }^{1}$ \\ Christian Bjørbaek, ${ }^{3}$ and Martin G. Myers Jr. ${ }^{1}$
}

\begin{abstract}
${ }^{1}$ Departments of Internal Medicine and Molecular and Integrative Physiology, University of Michigan Medical School, Ann Arbor, Michigan, USA. ${ }^{2}$ Department of Molecular Medicine and Surgery, Karolinska Institutet, Stockholm, Sweden. ${ }^{3}$ Department of Medicine, Beth Israel Deaconess Medical Center, Boston, Massachusetts, USA.
\end{abstract}

\begin{abstract}
The adipose-derived hormone, leptin, acts via its receptor (LRb) to convey the status of body energy stores to the brain, decreasing feeding and potentiating neuroendocrine energy expenditure. The failure of high levels of leptin in most obese individuals to promote weight loss defines a state of diminished responsiveness to increased leptin, termed leptin resistance. Leptin stimulates the phosphorylation of several tyrosine residues on $L R b$ to mediate leptin action. We homologously replaced LRb in mice with a receptor with a mutation in one of these sites (Tyr985) in order to examine its role in leptin action and signal attenuation in vivo. Mice homozygous for this mutation are neuroendocrinologically normal, but females demonstrate decreased feeding, decreased expression of orexigenic neuropeptides, protection from high-fat diet-induced obesity, and increased leptin sensitivity in a sex-biased manner. Thus, leptin activates autoinhibitory signals via LRb Tyr985 to attenuate the anti-adiposity effects of leptin, especially in females, potentially contributing to leptin insensitivity in obesity.
\end{abstract}

\section{Introduction}

The prevalence of obesity continues to increase at alarming rates throughout the world, fostering the rise in obesity-related comorbidities, such as diabetes and cardiovascular disease. While body energy homeostasis is closely regulated, only recently have we begun to understand the physiologic mechanisms that regulate feeding and body weight to effect this balance. One important effector of body energy homeostasis is leptin, which is produced by adipocytes as a signal of the repletion of body energy stores. Leptin acts in the CNS to promote satiety and enable neuroendocrine energy expenditure (1-7). The lack of leptin action due to mutations in leptin (e.g., $o b / o b$ mice) or in the active (b) form of the leptin receptor (LRb; e.g., $d b / d b$ mice) or as a consequence of lowered fat stores results in increased appetite and an energy-sparing neuroendocrine starvation response that includes infertility and growth retardation $(3,8)$. In $o b / o b$ and $d b / d b$ animals, hyperphagia paired with decreased energy expenditure results in morbid obesity and a propensity to develop type 2 diabetes. Conversely, in normal leptinsensitive animals, high leptin levels tend to reduce appetite and permit neuroendocrine energy expenditure, and leptin administration decreases feeding and body weight while preserving metabolic energy utilization. The failure of elevated leptin levels to mediate weight loss in common forms of human obesity suggests the attenuation of leptin action (leptin resistance) in obese states, as with diet-induced obesity in rodents (9-11). Potential mechanisms to explain this leptin resistance include alterations in leptin transport into the CNS and inhibition of leptin signaling $(12,13)$.

Nonstandard abbreviations used: AgRP, agouti-related peptide; ARC, arcuate nucleus of the hypothalamus; HF diet, high-fat diet; Lepr, leptin receptor; LRb, active (b) form of the leptin receptor; NC diet, normal chow diet; NPY, neuropeptide Y; POMC, proopiomelanocortin; SHP2, Src homology-containing tyrosine phosphatase-2. Conflict of interest: The authors have declared that no conflict of interest exists. Citation for this article: J. Clin. Invest. 117:1354-1360 (2007). doi:10.1172/JCI30688.
Leptin binding activates the LRb-associated Jak2 tyrosine kinase to mediate tyrosine phosphorylation-dependent leptin signaling via several pathways (Figure 1A) (1, 14-17): phosphorylated LRb Tyr1138 recruits the latent transcription factor STAT3 to mediate its activation $(14,16,17)$, and Tyr1077 activates STAT5 during LRb signaling in cultured cells (18). Phosphorylated Tyr985 of LRb binds Src homology-containing tyrosine phosphatase-2 (SHP2, also known as PTPN11), which participates in ERK activation during leptin signaling in cultured cells $(16,19)$. Tyr985 additionally binds SOCS3 and contributes to the attenuation of LRb signaling in vitro $(16,19,20)$. The LRb-associated Jak2 may also mediate signals independently of LRb tyrosine phosphorylation sites $(1,16)$ as well as provide a second, lower-affinity binding site for SOCS3 $(21,22)$. The cellular mechanisms by which leptin and $\mathrm{LRb}$ regulate signaling via other effector systems such as PI3K, mammalian target of rapamycin (mTOR), and AMP-activated protein kinase (AMPK) pathways are not yet understood (23-25).

We have previously demonstrated that signals mediated by Tyr1138 of LRb are central to the regulation of feeding and energy expenditure by leptin, while Tyr1138-independent signals are important for the control of glucose homeostasis, growth, and reproduction by leptin $(26,27)$. In this report, we examine the contribution of Tyr985 to LRb function in vivo in order to determine whether Tyr985 mediates these Tyr1138-independent or other leptin actions. We also examine a role for Tyr985 in the attenuation of leptin signaling.

\section{Results}

In order to understand the contribution of LRb Tyr985 to leptin action and inhibition in vivo, we generated mice in which the LRbspecific exon $18 \mathrm{~b}$ of the leptin receptor gene (Lepr) was homologously replaced by a mutant exon $18 \mathrm{~b}$ containing a substitution of Tyr985 (Lepr ${ }^{\text {Len985 }}$, encoding LRb ${ }^{\text {L985 }}$ ) (Figure 1B). This Tyr $\rightarrow$ Leu mutation was employed to facilitate genotyping (it creates a novel 

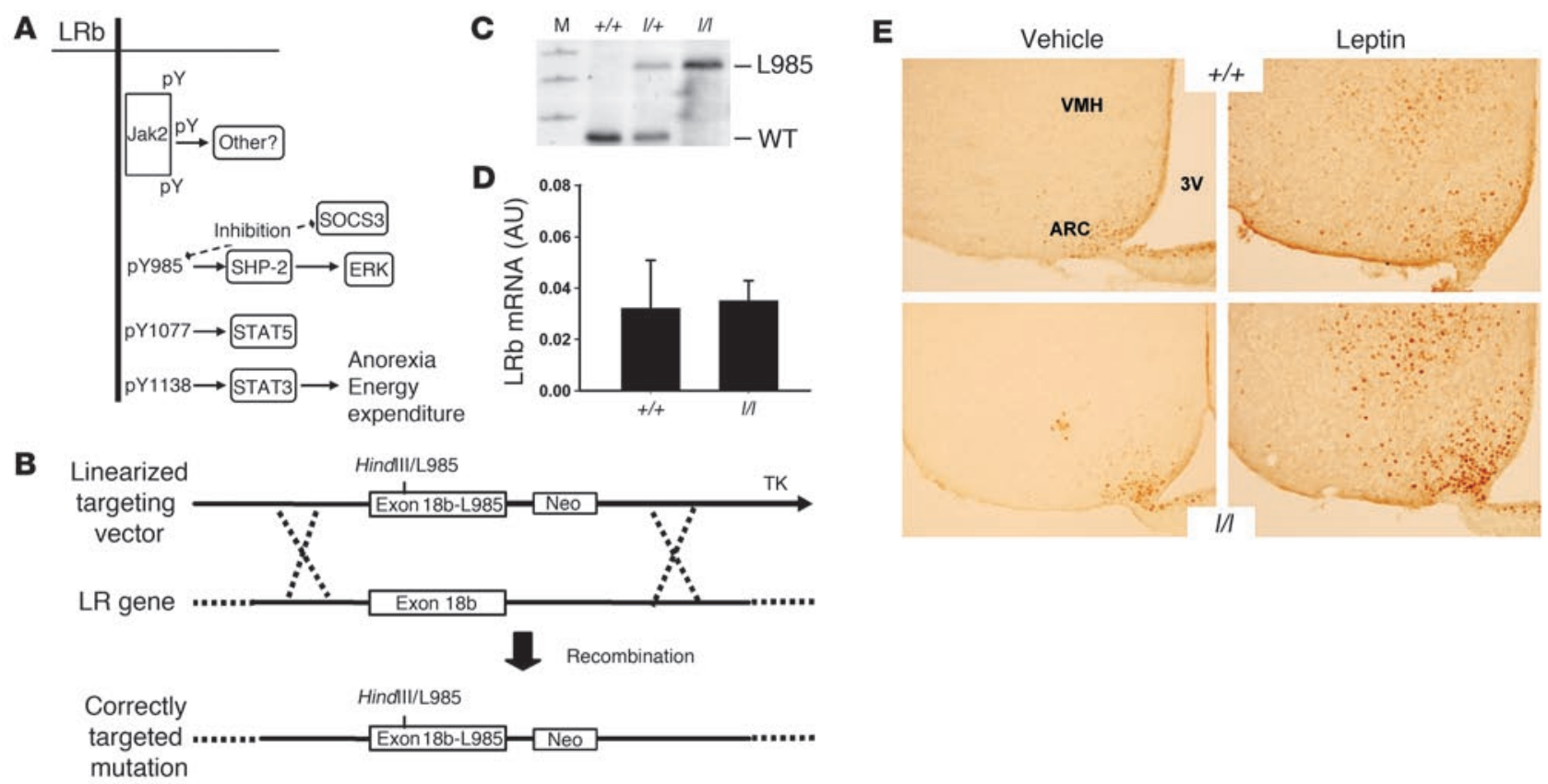

\section{Figure 1}

Generation of mice expressing LRb ${ }^{L 985}$. (A) Cartoon of signaling by LRb showing the activation of specific signals by individual tyrosine phosphorylation (pY) sites. (B) Targeting strategy for generation of the Lepr $r^{L 985}$ allele. Shown is the targeting vector containing the LRb-specific exon $18 \mathrm{~b}$ with the mutation of Tyr985 (which also generates a novel HindllI site) (exon18b-L985) plus Neo and thymidine kinase (TK) cassettes for positive and negative selection, respectively. Recombination in ES cells replaces the wild-type exon 18b with exon $18 \mathrm{~b}$-L985 and the Neo cassette, to mediate physiologic expression of the mutant LRb from the endogenous Lepr gene, as previously described (26). (C) Southern blot using a Lepr locus probe, demonstrating correct targeting of the locus in +/+, I/+, and I// mice. M, marker. (D) qPCR for hypothalamic LRb mRNA expression in +/+ and I// mice. (E) Hypothalamic detection of STAT3(PY) in +/+ and I// mice. Age- and sex-matched animals were treated i.p. with vehicle or leptin for 30 minutes. Brains were processed for immunohistochemical detection of STAT3(PY) in the hypothalamus; representative sections showing the $\mathrm{ARC}$ and ventromedial hypothalamic nucleus $(\mathrm{VMH})$ are shown. For reference, position of the third cerebral ventricle (3V) is shown. Original magnification, $\times 20$.

HindIII site), abrogate phosphorylation of the site, and block SHP2/SOCS3 recruitment, as demonstrated by the extensive study of this mutation in cultured cells $(16,19,20)$. This gene-targeting strategy expresses mutant LRb molecules from the genomic context of endogenous LRb so that expression patterns and levels of mutant LRb mirror those of wild-type LRb (26). Heterozygous Lepr $^{\text {Len } 985} /+(l /+)$ animals were intercrossed to generate $+/+, l /+$, and homozygous $(l / l)$ littermates for study (Figure $1 C$ ). We confirmed normal hypothalamic expression of leptin receptor mRNA in $l / l$ mice by quantitative real-time RT-PCR (Figure 1D).

In order to determine the distribution and function of $\mathrm{LRb}^{\mathrm{L} 985}$ in vivo, we examined leptin-stimulated signaling by analyzing the phosphorylation of STAT3 (pSTAT3) in the basomedial hypothalamus by immunohistochemistry (Figure 1E). As expected, +/+ and $l / l$ mice demonstrated similar patterns of leptin-stimulated STAT3 activation, confirming the normal distribution of functional $\mathrm{LRb}^{\mathrm{L} 985}$ in $l / l$ mice.

We initially examined the regulation of body weight in $+/+, l /+$, and $l / l$ littermates on the segregating $(C 57 ; 129)$ background fed a high-calorie breeder chow diet (Figure 2 ). The body weight of $l /+$ and $l / l$ animals of each sex were lower than for $+/+$ animals (Figure 2, A and B); while these differences did not reach statistical significance for male $l / l$ animals or $l /+$ animals of either sex, the body weight of $l / l$ females was decreased significantly at older ages compared with that of $+/+$ females. This difference in body weight corresponded to a significant decrease in the weight of gonadal fat pads in $l / l$ females only (Figure $2, \mathrm{C}$ and F). Circulating leptin levels were decreased in $l / l$ animals of both sexes compared with those of $+/+$ animals, however (Figure 2, D and G), suggesting the possibility of more modest affect of Tyr985 mutation on body adiposity in males. Insulin levels, while not different in males, were significantly decreased in $l / l$ females (Figure 2, E and $\mathrm{H}$ ), suggesting potentially increased insulin sensitivity in lean $l / l$ females. Overall, these data suggest that mutation of Tyr985 of LRb in vivo results in decreased adiposity, especially in females, consistent with the notion of increased leptin action in these animals.

To investigate susceptibility to diet-induced obesity in $l / l$ animals, we examined energy homeostasis in C57BL/6 l/l, l/+, and +/+ animals on normal chow (NC) and high-fat (HF) diets beginning at 5 weeks of age (Figure 3, A-D). On this background, body weights of $l / l$ and $l /+$ animals did not differ from age- and sex-matched $+/+$ animals, with the exception of older females fed HF chow, which were significantly lighter than $+/+$ and $l /+$ animals. Indeed, while weight gain over the course of the study was not different between $+/+$ and $l /+$ animals, $l / l$ and $+/+$ males on any diet, or $l / l$ and $+/+$ females on the NC diet, $l / l$ females on the HF diet exhibited decreased weight gain over the course of the study (Table 1). While food intake did not differ by genotype in males on any diet (Supplemental Figure 1; supplemental material available online with this article; doi:10.1172/JCI30688DS1) or on NC diet for females, both $l / l$ and 

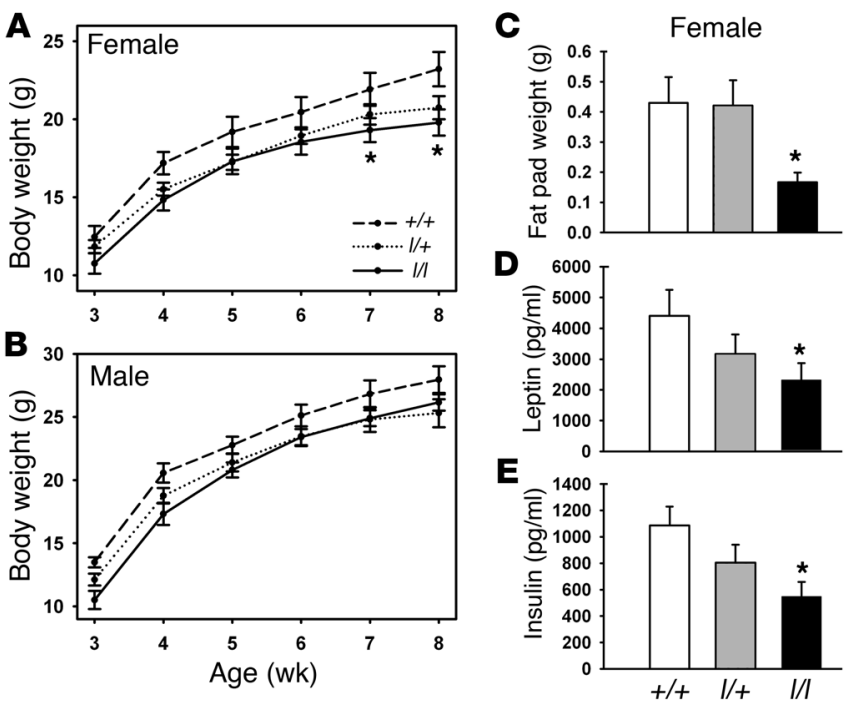

$l /+$ females demonstrated decreased food intake compared with +/+ animals at the inception of HF feeding (Figure 3, E and F).

These data demonstrate that the absence of LRb Tyr985 in females decreases body weight and also serves to decrease intake of palatable HF diets, suggesting that measures of adiposity should also be decreased in $l / l$ females. Indeed, fat pad weight was decreased in $l / l$ compared with that of $+/+$ females on either diet, and this decreased adiposity was reflected in decreased levels of circulating leptin in the $l / l$ females, even those on the NC diet - suggesting decreased adiposity in $l / l$ females, even in the absence of detectable alterations in total body weight on this diet (Figure 4, A and B). Neither adipose tissue weight nor leptin levels were different between genotypes in males (Supplemental Figure 1). Thus, $l / l$ females demonstrate decreased adiposity compared with +/+ mice on both outbred and inbred backgrounds, especially when exposed to a palatable, calorically dense diet. Circulating insulin levels in ad libitum-fed animals were also decreased in young $l / l$ and $l /+$ females as well as in older $l / l$ animals on the HF diet (Figure 4C), consistent with appropriately increased insulin sensitivity with decreased adiposity in $l / l$ females. No difference in insulin levels was observed between male $l / l$ and $+/+$ animals (Supplemental Figure 1).

\section{Figure 3}

Decreased body weight and feeding in $I / /$ females during HF feeding. (A-D) C57BL/6 female (A and B) and male (C and $\mathbf{D})+/+, / /+$, and $/ / /$ animals were provided NC (A and $\mathbf{C}$ ) or HF (B and $\mathbf{D}$ ) diets from the age of 5 weeks and weighed weekly until 12 weeks of age. All values represent mean \pm SEM. ANOVA and repeated measures ANOVA, $P=$ NS, except for $\# P<0.02$ for $I / /$ versus other groups where indicated. (E) Weight gained per female mouse from 5-12 weeks of age on each diet. (F) Food intake for first week of divergent diet for female mice of the indicated genotype. ${ }^{*} P<0.05$, ${ }^{* *} P<0.01$ versus $+/+$ animals, by ANOVA.

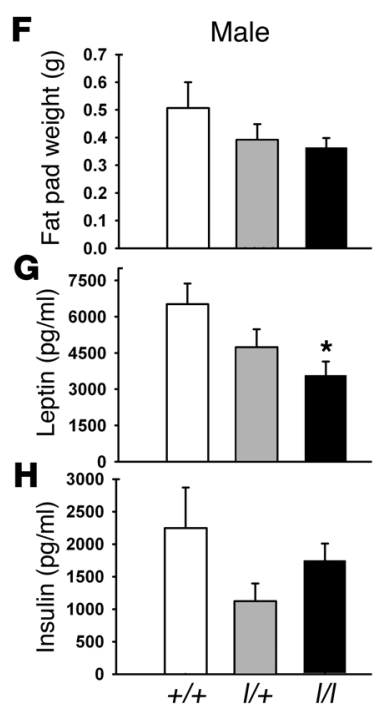

Figure 2

Leanness in I/I mice. (A and B) Mice of the indicated sex and genotype were weighed weekly from the time of weaning until the age of 8 weeks. No difference between curves by repeated measures ANOVA; ${ }^{*} P<0.05, I / I$ versus $+/+$ animals for pairwise comparisons (ANOVA) at indicated age. (C-H) Data regarding adipose tissue weight and hormone levels from 8-week-old female $+/+$ and $/ / /$ mice from $\mathbf{A}$ and $\mathbf{B}$. (C-E) Female mice. $(\mathbf{F}-\mathrm{H})$ Male mice. All values represent mean \pm SEM. ${ }^{*} P<0.05$ versus $+/+$ mice, by ANOVA.

Metabolic rate, as determined by oxygen consumption, was not different between female $+/+$ and $l / l$ animals (Supplemental Figure 2), however the finding of decreased measures of adiposity in $l / l$ animals on $\mathrm{NC}$ diet in the absence of measurable changes in food intake or energy expenditure may result from errors inherent to the measurement of food intake (e.g., chewing of less-palatable NC) or, alternatively, could result from altered partitioning of nutrients in the $l / l$ animals. The decreased feeding and normal metabolic rate of $l / l$ females in the face of decreased adiposity and lower leptin levels suggest increased leptin sensitivity of $l / l$ animals.
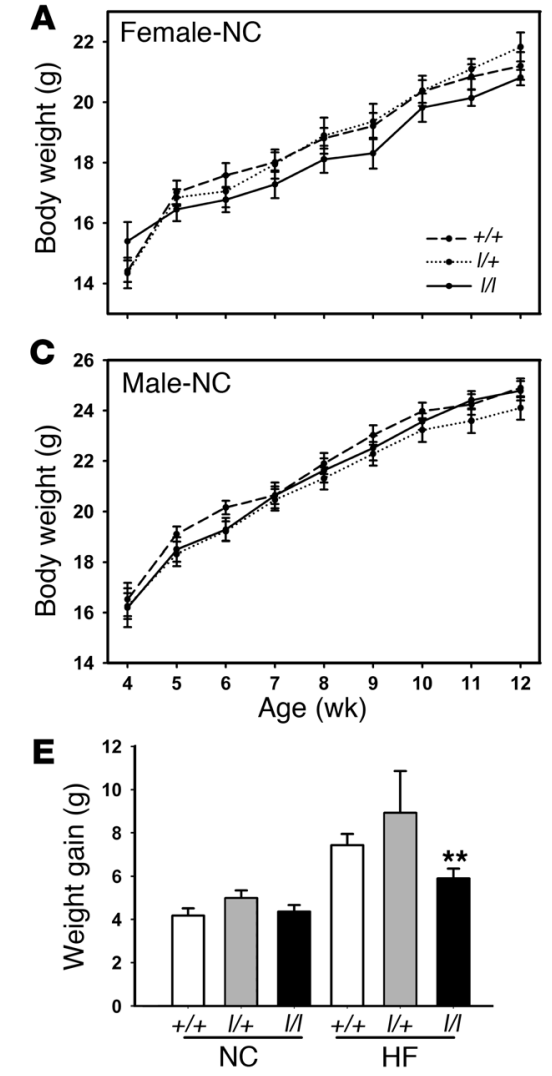
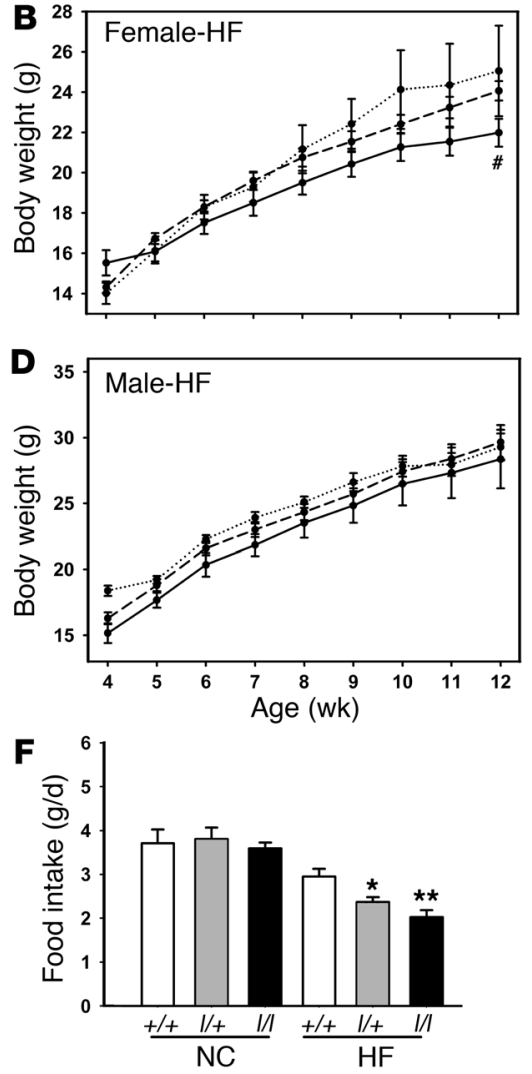
Table 1

Body weight and weight gain by C57BL/6 animals of all genotypes on NC and HF diets

$\begin{array}{lccccc}\text { Sex } & \text { Genotype } & \text { Diet } & \text { Weight } \mathbf{5} \text { wk (g) } & \text { Weight } \mathbf{1 2} \text { wk } \mathbf{( g )} & \text { Weight gain 5-12 wk (g) } \\ \text { Female } & +/+ & \text { NC } & 17.0 \pm 0.4 & 21.2 \pm 0.5 & 4.2 \pm 0.3 \\ & \text { I/+ } & \text { NC } & 16.8 \pm 0.3 & 21.8 \pm 0.5 & 5.0 \pm 0.4 \\ & \text { I/I } & \text { NC } & 16.5 \pm 0.4 & 20.8 \pm 0.3 & 4.4 \pm 0.3 \\ & +/+ & \text { HF } & 16.7 \pm 0.3 & 24.1 \pm 0.5^{\mathrm{A}} & 7.4 \pm 0.5^{\mathrm{A}} \\ & \text { I/+ } & \text { HF } & 16.1 \pm 0.5 & 25.1 \pm 2.0^{\mathrm{A}} & 8.9 \pm 1.9^{\mathrm{A}} \\ & \text { I/ } & \text { HF } & 16.1 \pm 0.6 & 22.0 \pm 0.7^{\mathrm{A}, \mathrm{B}} & 5.9 \pm 0.4^{\mathrm{A}, \mathrm{B}} \\ \text { Male } & +/+ & \text { NC } & 19.1 \pm 0.3 & 24.9 \pm 0.4 & 5.8 \pm 0.3 \\ & \text { I/+ } & \text { NC } & 18.3 \pm 0.5 & 24.1 \pm 0.5 & 5.7 \pm 0.4 \\ & \text { I/I } & \text { NC } & 18.5 \pm 0.5 & 24.8 \pm 0.4 & 6.3 \pm 0.4 \\ & +/+ & \text { HF } & 18.8 \pm 0.5 & 29.7 \pm 1.3^{\mathrm{A}} & 10.9 \pm 1.0^{\mathrm{A}} \\ & \text { I/+ } & \text { HF } & 19.2 \pm 0.3 & 29.3 \pm 1.0^{\mathrm{A}} & 10.1 \pm 1.2^{\mathrm{A}} \\ & \text { I/I } & \text { HF } & 17.7 \pm 0.6 & 28.4 \pm 2.2^{\mathrm{A}} & 9.7 \pm 1.4^{\mathrm{A}}\end{array}$

Data shown are mean \pm SEM. ${ }^{A} P<0.001$, compared with NC-fed animals, in group and pairwise comparisons, by ANOVA. ${ }^{\mathrm{B} P}<0.05$ compared with sex- and diet-matched $+/+$ animals, by ANOVA.

on body weight in $+/+$ animals $(17.1 \pm 0.3 \mathrm{~g}$ before treatment, $16.8 \pm 0.3 \mathrm{~g}$ following leptin treatment; $P=\mathrm{NS}$ ). In female $l / l$ mice, however, treatment resulted in a more than $5 \%$ decrease in overall body weight $(16.3 \pm 0.3 \mathrm{~g}$ before treatment, $15.2 \pm 0.3 \mathrm{~g}$ following leptin treatment; $P<0.001$ versus $+/+$ mice) and decreased feeding by approximately 1.5 -fold compared with $+/+$ animals (food intake prior to leptin treatment, $4.5 \pm 0.2 \mathrm{~g} / \mathrm{d}$ for $+/+$ mice, $4.5 \pm 0.1 \mathrm{~g} / \mathrm{d}$ for $l / l$ mice; during leptin treatment, $3.4 \pm 0.2 \mathrm{~g} / \mathrm{d}$ for $+/+$ mice, $2.8 \pm 0.2 \mathrm{~g} / \mathrm{d}$ for $l / l$ mice $[P<0.001]$; following leptin treatment, $4.6 \pm 0.2 \mathrm{~g} / \mathrm{d}$ for $+/+$ mice, $4.7 \pm 0.2 \mathrm{~g} / \mathrm{d}$ for $l / l$ mice) . Thus, female $l / l$ animals are more sensitive to leptin than are +/+ animals, suggesting that Tyr985 inhibits leptin action in vivo.

Indeed, the baseline pSTAT3 in the arcuate nucleus of the hypothalamus (ARC), which reflects signaling by endogenous leptin via $\mathrm{LRb}$, tended to be higher in $l / l$ compared with $+/+\mathrm{ARC}$ tissues and was significantly increased in $l / l$ compared with $+/+$ animals on NC diet (Figure 4D), even though circulating levels of leptin were lower in $l / l$ animals - indicating increased leptin sensitivity of signaling by $\mathrm{LRb}^{\mathrm{L} 985}$ compared with $\mathrm{LRb}$ in vivo $(20,28,29)$.

An important component of leptin action is mediated by the ARC, where leptin promotes the expression and secretion of appetite-suppressing (anorectic) neuropeptides derived from the proopiomelanocortin (POMC) precursor and inhibits the production and release of the orexigenic peptides neuropeptide Y (NPY) and agouti-related peptide (AgRP) (2). In order to determine whether the lean phenotype of $l / l$ females may result in part from enhanced leptin action in these ARC neurons, we examined neuropeptide mRNA expression in hypothalami from $+/+$ and $l / l$ females fed $N C$ and $\mathrm{HF}$ diets (Figure 5, A-C). While POMC mRNA levels demonstrated no consistent alteration among groups, both AgRP and NPY mRNA levels tended to be lower in the $l / l$ mice and were significantly lower in $l / l$ animals on the HF diet compared with $+/+$ animals on the HF $\operatorname{diet}(P<0.05)$, consistent with the prediction of increased sensitivity to leptin in $l / l$ animals. We also examined mRNA expression for the binding partners of Tyr985 (SOCS3 and SHP2), which were not altered in $l / l$ compared with $+/+$ animals on either diet (Figure 5, D and E).

In order to directly compare leptin sensitivity in $+/+$ and $l / l$ animals, we examined the physiological response to low-dose leptin treatment in 6-week-old NC-fed $l / l$ and $+/+$ females (Figure 6). In this paradigm, the amount of leptin administered had little effect

\section{Figure 4}

Decreased measures of adiposity and increased hypothalamic leptin action in female I/I animals. (A and $\mathbf{C}$ ) Serum leptin and insulin levels from female mice from Figure 3 at 4 and 10 weeks of age. (B) Weight of perigonadal fat pads from animals in Figure 3 at time of sacrifice. (D) At the age of 12 weeks, the ARC was dissected for the detection of STAT3(PY) (representative data shown in inset) and total STAT3 by immunoblotting. The ratio of STAT3(PY) normalized for total STAT3 is plotted for each group of animals. Data are normalized to levels in NC $+/+$ animals, which were set as $100 \%$. All values represent mean \pm SEM. ${ }^{*} P<0.05$ versus $+/+$ animals of same age and diet, by ANOVA.
Our previous finding of hyperphagia and obesity but preserved reproductive and growth function in mice lacking LRb Tyr1138/ STAT3 signals suggests that LRb mediates control of the reproductive and growth axes by signals other than Tyr1138/STAT3 $(26,30)$. The C57BL/ $6 \mathrm{l} / \mathrm{l}$ males and females were also fertile and displayed normal growth, suggesting that signals mediated by Tyr 985 are not required for the stimulation of the growth and reproductive axes by leptin (Table 2). In addition, the finding of appropriately decreased circulating insulin levels in lean normoglycemic $l / l$ mice also suggests appropriate regulation of insulin sensitivity independently of Tyr985 (Figures 2 and 4). Glucose levels in ad libitum-fed animals did not vary among genotypes on any diet (data not shown).

We furthermore confirmed that $l / l$ mice are neuroendocrinologically different from $d b / d b$ animals by interbreeding $l /+$ and $d b /+$ animals to generate (fertile) $d b / l$ animals, which were then interbred to obtain littermate $d b / d b$ and $l / l$ animals. Comparison of these animals confirmed the preservation of reproductive function and growth in $l / l$ relative to $d b / d b$ animals (Supplemental Table 1 ).

\section{Discussion}

Mutation of Tyr985 in vivo results in reduced feeding and adiposity, decreased orexigenic ARC neuropeptide expression, and
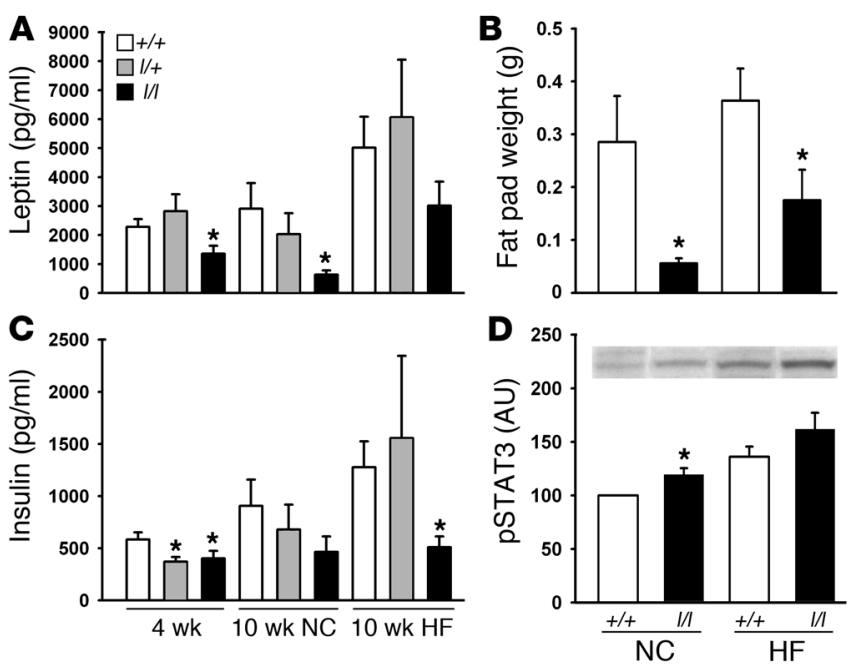

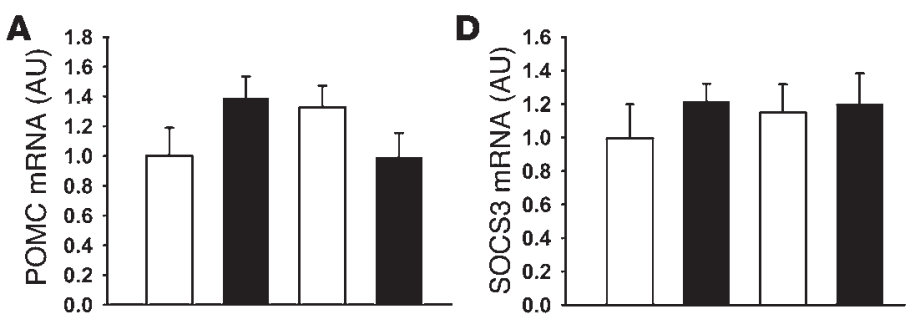

B
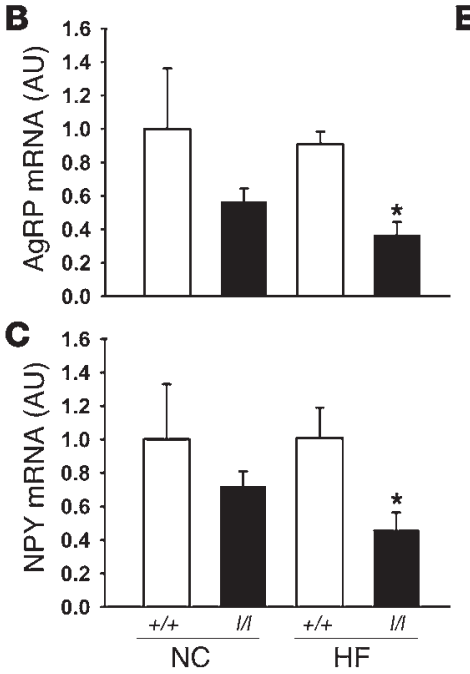

\section{E}

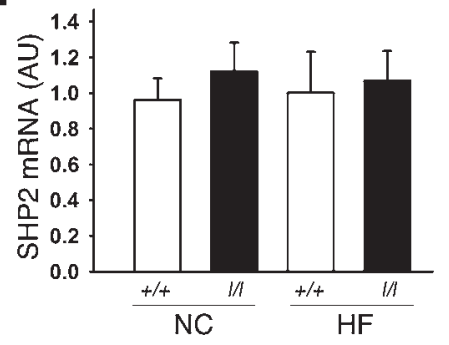

\section{Figure 5}

ARC neuropeptide and Tyr985-interacting protein gene expression in I/I females. ARC mRNA levels for (A) POMC, (B) AgRP, (C) NPY, (D) SOCS3, and (E) SHP2 from animals in Figure 3. Data are normalized to levels in $\mathrm{NC}+/+$ animals, which were set as $100 \%$. All values represent mean \pm SEM. ${ }^{*} P<0.05$ versus $+/+$ animals of same age and diet, by ANOVA.

diet-induced obesity) is reminiscent of results for deletion of the Tyr985-binding inhibitor of leptin signaling, SOCS3, but contrasts with the effect of PTP1B knockout (32-35). PTP1B, another important physiologic inhibitor of leptin and insulin action, is not appreciably increased by leptin treatment, and mice null for this molecule demonstrate increased leanness on both NC and HF diets, with the effect being more prominent on the NC diet $(34,36)$.

Since Tyr985 of LRb recruits both SHP2 and SOCS3 $(20,28$, 29), the failure of $\mathrm{LRb}^{\mathrm{L} 985}$ to recruit either of these proteins could theoretically underlie the lean, leptin-sensitive phenotype of $l / l$ mice. Most data from cultured cells and animals support a primary role for SOCS3 in the inhibition of LRb signaling, however $(19-21,32,35,37,38)$. SOCS3 mRNA expression was similar between $+/+$ and $l / l$ mice, suggesting the effect of LRb Tyr985 in the attenuation of leptin action in vivo is not due to differences in SOCS3 expression between $+/+$ and $l / l$ animals. Since leptin treatment increases STATdependent SOCS3 expression, the finding that SOCS3 mRNA expression is not increased in $l / l$ females with enhanced baseline STAT3 signaling is consistent with the possibility that factors other than leptin may represent the main regulators of hypothalamic SOCS3 expression under physiologic conditions.

While female $l / l$ animals consistently display a leptin-sensitive and lean phenotype, male $l / l$ animals display remarkably little of this effect. The female predominance of the lean hypophagic $l / l$ phenotype is an intriguing and unexpected result from this study. These results are consistent with the finding of a similarly more robust leptin-sensitive phenotype for SOCS3-deficient females than males, however $(32,35)$. While the mechanism for this sex specificity remains unclear, these findings are consistent with the observation that the regulation of feeding in females is more attenuation of LRb signaling thus represents a leptin-stimn autoinhibitory signal, the magnitude of which would be predicted to increase in proportion to leptin levels and thus more profoundly attenuate the amplitude of the LRb signal at high leptin levels, as found in obesity. Consistent with this, our data demonstrate a more robust divergence of feeding and body weight between $+/+$ and $l / l$ animals fed HF diet or breeder chow than in $+/+$ and $l / l$ animals fed NC diet. Indeed, while weight gain over the course of the NC feeding study was similar in $+/+$ and $l / l$ animals, the increased weight gain attributable to HF diet (weight gained on NC diet subtracted from weight gained on HF diet) was 3.2 $\mathrm{g}$ for $+/+$ females, but only $1.5 \mathrm{~g}$ for $l / l$ females. Hence, at lower leptin levels in lean animals fed NC diet, the inhibitory function of Tyr985 on leptin action appears to be relatively modest but becomes more pronounced with increased adiposity and higher circulating leptin levels. The action of Tyr985 thus provides one reasonable mechanism to explain the leptin resistance associated with obesity. The more pronounced effect of Tyr985 mutation in the face of obesity-predisposing, high-leptin states (such as
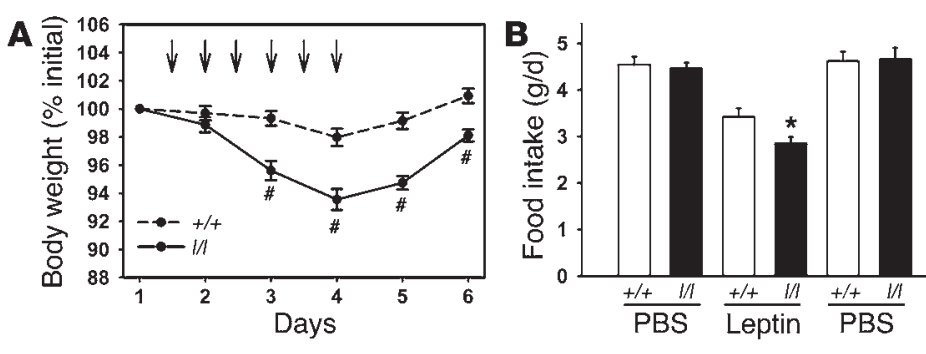

\section{Figure 6}

Increased in vivo leptin sensitivity in I// mice. Seven-week-old female +/+ and $I / /$ mice fed NC were injected i.p. twice daily with PBS for 3 days, then with leptin for 3 days, and then with PBS for 2 days. (A) Body weight was measured once per day during the injection period. Day 1 represents the average weight during the initial (first 3 days) PBS injections. Arrows indicate times of leptin injections. (B) Average 24-hour food intake during injection periods. All values represent mean \pm SEM. ${ }^{*} P<0.05$ and ${ }^{\#} P<0.001$ versus $+/+$ mice. 
Table 2

Reproductive and growth phenotype of C57BL/6 +/+ and I//animals on NC diet

\begin{tabular}{|c|c|c|c|c|}
\hline \multirow[b]{2}{*}{ Genotype } & \multicolumn{2}{|c|}{ Male } & \multicolumn{2}{|c|}{ Female } \\
\hline & +/+ & 1/I & $+/+$ & $\mathrm{l} / \mathrm{I}$ \\
\hline Fertility (reproduced/tested) & 9/9 & $11 / 11$ & $11 / 11$ & $12 / 12$ \\
\hline Lean mass ( $\mathrm{g}$ ) & $22.7 \pm 0.6$ & $23.4 \pm 0.8$ & $18.8 \pm 0.4$ & $19.0 \pm 0.3$ \\
\hline Fat mass $(\mathrm{g})$ & $4.6 \pm 0.2$ & $4.8 \pm 0.3$ & $4.1 \pm 0.3$ & $3.5 \pm 0.2$ \\
\hline Snout-anus length (mm) & $90.8 \pm 1.2$ & $93.1 \pm 0.8$ & $89.9 \pm 1.7$ & $89.8 \pm 0.7$ \\
\hline
\end{tabular}

Data are mean $\pm \mathrm{SEM}$. For all $/ / /$ animals, $P=\mathrm{NS}$ versus $+/+$ animals, by unpaired 2-tailed Student's $t$ test.

responsive to leptin, while that in males is more responsive to insulin $(39,40)$ - which predicts that specific sensitization of leptin signaling would result in a stronger phenotype in females than in males. Indeed, estrogen interacts with the same neural and signaling apparatus as leptin in the hypothalamus to regulate body energy homeostasis (41). Determining the precise roles for various sex-specific factors, such as gonadal hormones, in the sex specificity of the $l / l$ phenotype will require additional study.

Mice of either sex heterozygous for mutation of Tyr985 (l/+ mice) display relatively little of the lean, leptin-sensitive phenotype demonstrated by the homozygous $l / l$ females. Most measures of energy balance in the $l /+$ female mice trend toward a phenotype intermediate between that of the $+/+$ and the $l / l$ animals, suggesting that, while the effect of Tyr985 mutation is not dominant, a $50 \%$ reduction in this pathway may predispose toward leanness, consistent with codominance as for heterozygosity for SOCS3 (32). Thus, it is possible that variability of the Tyr $985 /$ SOCS3 pathway in large, heterogeneous populations of genetically diverse individuals (such as humans) could meaningfully impact energy balance. At a molecular level, this also suggests that the amplitude of LRb signaling is regulated by the relative amount of SOCS3 within the LRb dimer or oligomer and that SOCS3 levels within the LRb signaling complex may roughly approximate the amount of phosphorylated Tyr985.

While it has become increasingly apparent that actions outside the ARC mediate important aspects of the leptin response and contribute to the regulation of body energy homeostasis $(42,43)$, ARC neurons remain a crucial site of leptin action, and the finding of decreased orexigenic ARC neuropeptide mRNA levels in $l / l$ mice suggests that increased leptin sensitivity in these animals operates at least in part in these specific neurons of the ARC. While the unaltered POMC expression in the hypothalamus of $l / l$ mice might suggest some neuronal specificity to the phenotype of these mice, it is also possible that our inability to detect such changes is a consequence of the virtually maximal expression of this gene in fed animals, as were studied in this experiment. Although the effects of Tyr985 and other sensitizers of leptin signaling are most easily and commonly studied by examining the regulation of STAT3 signaling, the increased suppression of AgRP and NPY mRNA expression in the $l / l$ animals also suggests the importance of Tyr985 in attenuating STAT3-independent signals, since the regulation of these neuropeptides may be largely independent of STAT3 $(26,44,45)$.

The phenotype of $l / l$ mice also suggests that, while Tyr985 is required for SHP2 signaling, this signal is not required for the regulation of growth or reproduction by leptin and does not mediate essential anorectic signals. This finding contrasts with the finding of obesity and altered neuroendocrine function in animals with deletion of SHP2 in the forebrain (37), consistent with the notion that disruption of SHP2 alters signaling by numerous factors other than leptin, and in a wide variety of neuronal populations $(46,47)$. Since LRb Tyr1138/STAT3-independent signals contribute to the regulation of growth, reproduction, and glucose homeostasis by leptin (26), the LRb Tyr1077/STAT5 pathway or signals mediated by the LRb-associated Jak2 independently of LRb tyrosine phosphorylation $(18,24,30)$ are likely candidates to mediate the control of these neuroendocrine actions by leptin.

Overall, these results suggest that the inhibitory LRb Tyr985 signal may underlie the decreased incremental responsiveness to leptin observed in conditions of increased adiposity and leptin levels (the hallmark of leptin resistance), especially in females. This thus suggests a role for this feedback pathway in leptin resistance and the establishment or maintenance of common forms of obesity.

\section{Methods}

Animals. Interbreeding of $l /+$ mice yielded $+/+, l /+$, and $l / l$ mice for study at the expected Mendelian frequencies. Genotyping of ES cell clones was by Southern analysis; mice were initially genotyped as described for other LRb knock-in mice (26) and subsequently genotyped via SNP analysis by automated fluorescent real-time PCR (Applied Biosystems). $l /+$ animals were backcrossed to the $\mathrm{C} 57 \mathrm{BL} / 6$ background using a speed congenic protocol at Charles River Laboratories Inc. to greater than 95\% homozygosity (N4-N6 animals) before intercrossing to generate experimental C57BL/6 animals. $\mathrm{C} 57 \mathrm{BL} / 6$ and $\mathrm{C} 57 \mathrm{BL} / 6 \mathrm{db} /+$ mice for breeding were from The Jackson Laboratory. Mice were housed in accredited animal facilities of the Joslin Diabetes Center (Boston, Massachusetts, USA) and the University of Michigan with ad libitum access to chow and water. Mice for study were housed individually from the time of weaning at 21 days of age, with weekly monitoring of body weight. All procedures were approved by the Institutional Animal Care and Use Committee at Joslin Diabetes Center and the University of Michigan.

Leptin stimulation and immunohistochemical analysis of bypothalamic signaling. Eight- to ten-week-old mice were treated with recombinant mouse leptin $(5 \mu \mathrm{g} / \mathrm{g})$ (National Hormone \& Peptide Program) or vehicle i.p. for 30 minutes before being deeply anesthetized $(90 \mathrm{mg} / \mathrm{kg}$ sodium pentobarbital) and perfused transcardially with formalin. Removal of the brain, postfixation, cryoprotection, sectioning, and immunohistochemistry were as described in ref. 11. In brief, free-floating tissue sections were blocked in donkey serum and then incubated with antiphosphorylated STAT3 antibody $(1: 2,000)$ obtained from Cell Signaling Technology Inc. Sections were developed using diaminobenzidine, and images were captured under light microscopy with a digital camera.

Phenotypic data. Blood was collected for serum determination of insulin and leptin (Rat Insulin ELISA Kit and Mouse Leptin ELISA Kit; Crystal Chem Inc.) between $2 \mathrm{pm}$ and $4 \mathrm{pm}$. Snout-anus length was measured with a micrometer. Body composition was determined by dual-energyx-ray absorptiometry (DEXA) (PIXImus; Lunar). For HF diet-fed animals, $45 \mathrm{kcal} \%$ fat chow (catalog no. D12451; Research Diets Inc.) was provided from 5 weeks of age. Breeder chow was Purina Labdiet 5008. NC was Purina Labdiet 5001. Food consumption was determined by weighing the chow once every second day during the first week of HF feeding.

In vivo leptin sensitivity. Female $+/+$ and $l / l$ mice of 7 weeks of age were injected i.p. at 8 am and 4:30 pm with PBS or leptin $(1 \mathrm{mg} / \mathrm{kg})$ according to the following scheme: PBS injections for 3 days, followed by leptin injections for 3 days, followed by PBS injections for 2 days. Animals and chow were weighed daily during the injection period. 
Analysis of bypothalamic protein and RNA. Hypothalami were isolated from random-fed 8-week-old mice between $10 \mathrm{am}$ and $12 \mathrm{am}$, and ARC was microdissected and snap-frozen. Analysis of STAT3(PY) was as described in ref. 11. For other samples, total RNA was isolated using ULTRASPEC RNA reagent (BIOTECX Laboratories Inc.). For determination of relative RNA concentration, total hypothalamic RNA was subjected to automated fluorescent RT-PCR on an ABI Prism 7700 Sequence Detection System (Applied Biosystems). GAPDH control, SOCS3, and LRb primers and probes were as supplied by Applied Biosystems. POMC, AgRP, and NPY primers and probes were as previously described (26). Each predicted RT-PCR product spanned an intron/ exon junction. Each RT-PCR reaction was determined to be in the linear range for quantitation by comparison with serial dilutions of input hypothalamic or ARC RNA.

Statistics. Statistical analysis was performed by ANOVA or unpaired 2-tailed Student's $t$ test using the statistical software SigmaStat 3.0 (Systat Software Inc.) or ezANOVA (http://www.sph.sc.edu/comd/rorden/ ezanova/home.html). Analyses were within groups, except for some instances of repeated measures as noted in the text or figure legends.

\section{Acknowledgments}

Supported by grants from the NIH (DK56731 and DK57768 to M.G. Myers Jr.), AMF-sjukförsäkring Jubilée Foundation for Research in National Diseases, and Swedish Society for Medical Research (to M. Björnholm).

Received for publication October 19, 2006, and accepted in revised form February 6, 2007.

Address correspondence to: Martin G. Myers Jr., Division of Metabolism, Endocrinology and Diabetes, Department of Internal Medicine, University of Michigan Medical School, 4301 MSRB III, 1150 W. Medical Center Dr., Ann Arbor, Michigan 48109, USA. Phone: (734) 647-9515; Fax: (734) 936-6684; E-mail: mgmyers@umich.edu.
1. Myers, M.G., Jr. 2004. Leptin receptor signaling and the regulation of mammalian physiology. Recent Prog. Horm. Res. 59:287-304.

2. Elmquist, J.K., Coppari, R., Balthasar, N., Ichinose, M., and Lowell, B.B. 2005. Identifying hypothalamic pathways controlling food intake, body weight, and glucose homeostasis. J. Comp. Neurol. 493:63-71.

3. Ahima, R.S., et al. 1996. Role of leptin in the neuroendocrine response to fasting. Nature. 382:250-252.

4. Zhang, Y., et al. 1994. Positional cloning of the mouse obese gene and its human homologue. Nature. 372:425-432.

5. Chua, S.C., Jr., et al. 1996. Phenotypes of mouse diabetes and rat fatty due to mutations in the $\mathrm{OB}$ (Leptin) receptor. Science. 271:994-996.

6. Cohen, P., et al. 2001. Selective deletion of leptin receptor in neurons leads to obesity. J. Clin. Invest. 108:1113-1121. doi:10.1172/JCI200113914.

7. Kowalski, T.J., Liu, S.M., Leibel, R.L., and Chua, S.C., Jr. 2001. Transgenic complementation of leptin-receptor deficiency. I. Rescue of the obesity/ diabetes phenotype of LEPR-null mice expressing a LEPR-B transgene. Diabetes. 50:425-435.

8. Farooqi, I.S., and O'Rahilly, S. 2004. Monogenic human obesity syndromes. Recent Prog. Horm. Res. 59:409-424.

9. Mantzoros, C.S., and Flier, J.S. 2000. Editorial: leptin as a therapeutic agent - trials and tribulations. J. Clin. Endocrinol. Metab. 85:4000-4002.

10. El Haschimi, K., Pierroz, D.D., Hileman, S.M., Bjorbaek, C., and Flier, J.S. 2000. Two defects contribute to hypothalamic leptin resistance in mice with diet-induced obesity. J. Clin. Invest. 105:1827-1832.

11. Munzberg, H., Flier, J.S., and Bjorbaek, C. 2004. Region-specific leptin resistance within the hypothalamus of diet-induced-obese mice. Endocrinology. 145:4880-4889.

12. Banks, W.A. 2004. The many lives of leptin. Peptides. 25:331-338.

13. Munzberg, H., Bjornholm, M., Bates, S.H., and Myers, M.G., Jr. 2005. Leptin receptor action and mechanisms of leptin resistance. Cell. Mol. Life Sci. 62:642-652.

14. Baumann, H., et al. 1996. The full-length leptin receptor has signaling capabilities of interleukin 6type cytokine receptors. Proc. Natl. Acad. Sci. U. S. A. 93:8374-8378.

15. Tartaglia, L.A., et al. 1995. Identification and expression cloning of a leptin receptor, OB-R. Cell. 83:1263-1271.

16. Banks, A.S., Davis, S.M., Bates, S.H., and Myers, M.G., Jr. 2000. Activation of downstream signals by the long form of the leptin receptor. J. Biol. Chem.
275:14563-14572.

17. White, D.W., Kuropatwinski, K.K., Devos, R., Baumann, H., and Tartaglia, L.A. 1997. Leptin receptor (OB-R) signaling. J. Biol. Chem. 272:4065-4071.

18. Hekerman, P., et al. 2005. Pleiotropy of leptin receptor signalling is defined by distinct roles of the intracellular tyrosines. FEBS J. 272:109-119.

19. Bjorbaek, C., et al. 2001. Divergent roles of SHP-2 in ERK activation by leptin receptors. J. Biol. Chem. 276:4747-4755.

20. Bjorbaek, C., et al. 2000. SOCS3 mediates feedback inhibition of the leptin receptor via Tyr985. J. Biol. Chem. 275:40649-40657.

21. Dunn, S.L., et al. 2005. Feedback inhibition of leptin receptor/Jak2 signaling via Tyr1138 of the leptin receptor and suppressor of cytokine signaling 3. Mol. Endocrinol. 19:925-938.

22. Sasaki, A., et al. 2000. CIS3/SOCS3 suppresses erythropoietin signaling by binding the EPO receptor and JAK2. J. Biol. Chem. 275:29338-29347.

23. Minokoshi, Y., et al. 2004. AMP-kinase regulates food intake by responding to hormonal and nutrient signals in the hypothalamus. Nature. 428:569-574.

24. Niswender, K.D., et al. 2001. Intracellular signalling. Key enzyme in leptin-induced anorexia. Nature. 413:794-795.

25. Cota, D., et al. 2006. Hypothalamic mTOR signaling regulates food intake. Science. 312:927-930.

26. Bates, S.H., et al. 2003. STAT3 signaling is required for leptin regulation of energy balance but not reproduction. Nature. 421:856-859.

27. Bates, S.H., et al. 2004. LRb-STAT3 signaling is required for the neuroendocrine regulation of energy expenditure by leptin. Diabetes. 53:3067-3073.

28. Carpenter, L.R., Farruggella, T.J., Symes, A., Karow, M.L., and Yancopoulos, G. 1998. Enhancing leptin response by preventing SH2-containing phosphatase 2 interaction with Ob receptor. Proc. Natl. Acad. Sci. U. S. A. 95:6061-6066.

29. Li, C., and Friedman, J.M. 1999. Leptin receptor activation of $\mathrm{SH} 2$ domain containing protein tyrosine phosphatase 2 modulates Ob receptor signal transduction. Proc. Natl. Acad. Sci. U. S. A. 96:9677-9682.

30. Bates, S.H., and Myers, M.G., Jr. 2003. The role of leptin receptor signaling in feeding and neuroendocrine function. Trends Endocrinol. Metab. 14:447-452.

31. Vaisse, C., et al. 1996. Leptin activation of Stat3 in the hypothalamus of wild-type and $o b / o b$ mice but not $d b / d b$ mice. Nat. Genet. 14:95-97.

32. Howard, J.K., et al. 2004. Enhanced leptin sensitivity and attenuation of diet-induced obesity in mice with haploinsufficiency of Socs3. Nat. Med. 10:734-738.

33. Shi, H., Tzameli, I., Bjorbaek, C., and Flier, J.S. 2004. Suppressor of cytokine signaling 3 is a physiologic regulator of adipocyte Insulin signaling. J. Biol. Chem. 279:34733-34740.

34. Bence, K.K., et al. 2006. Neuronal PTP1B regulates body weight, adiposity and leptin action. Nat. Med. 12:917-924.

35. Mori, H., et al. 2004. Socs3 deficiency in the brain elevates leptin sensitivity and confers resistance to diet-induced obesity. Nat. Med. 10:739-743.

36. Zabolotny, J.M., et al. 2002. PTP1B regulates leptin signal transduction in vivo. Dev. Cell. 2:489-495.

37. Zhang, E.E., Chapeau, E., Hagihara, K., and Feng, G.S. 2004. Neuronal Shp2 tyrosine phosphatase controls energy balance and metabolism. Proc. Natl. Acad. Sci. U. S. A. 101:16064-16069.

38. Bjorbaek, C., Elmquist, J.K., Frantz, J.D., Shoelson, S.E., and Flier, J.S. 1998. Identification of SOCS-3 as a potential mediator of central leptin resistance. Mol. Cell. 1:619-625.

39. Clegg, D.J., Brown, L.M., Woods, S.C., and Benoit, S.C. 2006. Gonadal hormones determine sensitivity to central leptin and insulin. Diabetes. 55:978-987.

40. Woods, S.C., Gotoh, K., and Clegg, D.J. 2003. Gender differences in the control of energy homeostasis. Exp. Biol. Med. (Maywood). 228:1175-1180.

41. Gao, Q., et al. 2007. Anorectic estrogen mimics leptin's effect on the rewiring of melanocortin cells and Stat 3 signaling in obese animals. Nat. Med. 13:89-94.

42. Coppari, R., et al. 2005. The hypothalamic arcuate nucleus: a key site for mediating leptin's effects on glucose metabolism and locomotor activity. Cell Metab. 1:63-72.

43. Dhillon, H., et al. 2006. Leptin directly activates $\mathrm{SF} 1$ neurons in the $\mathrm{VMH}$, and this action by leptin is required for normal body-weight homeostasis. Neuron. 49:191-203.

44. Kitamura, T., et al. 2006. Forkhead protein FoxO1 mediates Agrp-dependent effects of leptin on food intake. Nat. Med. 12:534-540.

45. Kaelin, C.B., Xu, A.W., Lu, X.Y., and Barsh, G.S. 2004. Transcriptional regulation of agouti-related protein (Agrp) in transgenic mice. Endocrinology. 145:5798-5806.

46. Feng, G.S. 1999. Shp-2 tyrosine phosphatase: signaling one cell or many. Exp. Cell Res. 253:47-54.

47. Keilhack, H., David, F.S., McGregor, M., Cantley, L.C., and Neel, B.G. 2005. Diverse biochemical properties of Shp2 mutants. Implications for disease phenotypes. J. Biol. Chem. 280:30984-30993. 